\title{
DOUBLING STRATEGIES FOR BACKGAMMON-LIKE GAMES
}

\author{
E. O. TUCK
}

(Received 19 April 1979)

(Revised 4 September 1979)

\begin{abstract}
Optimal strategies are obtained for two-player games with an alternating stakedoubling option. A complete two-parameter analysis is provided for games that must end within two moves, and a recursive procedure then enables a solution for games of any number of moves. Examples are given of relevance to extreme end games in backgammon.
\end{abstract}

\section{Introduction}

We consider a two-player perfect-information game (of which backgammon is the model) in which either player has the right, immediately prior to moving, to demand that the stake be doubled. His opponent may accept and play on at the doubled stake, or quit, losing the previous (undoubled) stake. If the opponent accepts, he alone has the right to next double the stake, that is, to redouble, and so on.

The 20th-century introduction of this doubling feature into the ancient game of backgammon has been largely responsible for its recent rise in popularity. The main effect is to place a premium on the ability to estimate accurately the state of the game, in order to allow rational judgements to be made about offering or accepting doubles. This extra measure of skill is a valuable addition to a game which otherwise depends perhaps too much on the luck of the dice.

The analysis presented in the following Sections $2-4$ is intended to provide a framework for determination of optimal doubling strategies in a general class of games with this doubling feature, and is not necessarily restricted to backgammon. However, in Section 5 we give specific applications to some close end games in backgammon. These applications are all such that contact has been broken and a gammon is not possible, which are characteristics of most end games in back- 
gammon that are reflected in properties assumed in the analysis for general games.

The analysis can indeed proceed on a very general basis if we assume as in Section 3 that the game must end after at most one move by each player. All such games can be specified in terms of the two independent probabilities $(p, q)$ of winning with that single move, $p$ being the probability for the player who is to move first. We are able to provide complete strategies for such games, in the form of a diagram in the $(p, q)$ plane indicating regions in which either (i) no action should be taken or (ii) a double should be offered and accepted or (iii) a double should be offered and refused. In fact, region (ii) has a subregion in which, although a first double should be offered, a redouble (that is, a double that can only be offered by the player about to move) should not be offered.

The formulation of Section 4 enables analysis of games of any number of moves, but now we must be prepared to specify actual positions in the game, and a matrix of transition probabilities from one position to another. The analysis then determines expected profits for various (not necessarily optimal) strategies at the current position, in terms of (assumed known) profits and optimal strategies for positions closer to the end of the game. Comparison of these expectations then enables determination of the optimal strategies at the current position, and hence for all positions, in a recursive manner.

The specific application to backgammon end games in Section 5 confirms most accepted strategies (for example, as described in teaching books on that game, such as [1]), but indicates a few surprises at the borderlines where the strategies are changing. However, the difference in expected profit if the wrong decision is made in these borderline cases is minuscule.

\section{Expected profit functions}

We need to define the following expectation functions. Each $E_{n}, n=0$ to 5 , is a net expected profit to the player $A$ who is about to move, when the initial stake is $\$ 1$, under the outlined circumstances. Both players $A$ and $B$ are assumed to be using optimal strategies, except where specified.

$E_{0}$ : Neither player is ever permitted to double.

$E_{1}$ : Either player can make the first double.

$E_{2}$ : Only $A$ can make the first double.

$E_{3}$ : Only $B$ can make the first double.

$E_{4}$ : Either player can make the first double, but $A$ chooses not to do so.

$E_{5}$ : Only $A$ can make the first double, and chooses not to do so.

The expectation $E_{0}$ is naturally of no interest in analysis of doubling strategies, but may serve as a base value; it is of importance in some competitive circumstances, as in backgammon when the "Crawford rule" ([1], page 181) is in effect. 
Note that $E_{1}$ must take one of the three values $\left\{1,2 E_{3}, E_{4}\right\}$ and $E_{2}$ must take one of the three values $\left\{1,2 E_{3}, E_{5}\right\}$. Thus, if $A$ does offer the double, then either $B$ quits, so $E_{1}=1$, or $B$ accepts, thereby owning the sole right to the next double (that is, to a redouble) in a game at twice the stake, so that $E_{1}=2 E_{3}$. Hence $B$ 's optimal strategy is to accept if $2 E_{3}<1$ and to quit otherwise, thereby minimizing $A$ 's expected profit.

Similariy, $A$ 's optimal strategy (maximizing his own profit) is to offer the double if the resultant expectation when $B$ is using an optimal strategy, namely $\min \left\{1,2 E_{3}\right\}$, exceeds the expectation $E_{4}$ or $E_{5}$ when the double is not offered. In fact, whenever a double by $A$ is not certain to be offered, we must have

$$
\min \left\{1,2 E_{3}\right\}=2 E_{3} \text {. }
$$

This is because (in the absence of devices such as gammons for winning more than the initial stake in an undoubled game) $A$ must always double if he knows that $B$ will quit; after all, $\$ 1$ for sure is better than $\$ 1$ maybe!

Thus $A$ 's optimal strategy is to offer a non-exclusive double if $2 E_{3}>E_{4}$, and to offer a double that $A$ alone can offer (that is, a redouble) if $2 E_{3}>E_{5}$. The expected profits when this optimal strategy is in operation are therefore

and

$$
E_{1}=\max \left\{E_{4}, \min \left(1,2 E_{3}\right)\right\}
$$

$$
E_{2}=\max \left\{E_{5}, \min \left(1,2 E_{3}\right)\right\} \text {. }
$$

We should also note that $E_{5} \geqslant E_{4}$, since the exclusive right to double is of nonnegative value to $A$. Thus there are only three types of strategy available to $A$, namely:

(i) take no action, if $2 E_{3}<E_{4}$;

(ii) double but do not redouble, if $E_{4}<2 E_{3}<E_{5}$;

(iii) double or redouble, if $2 E_{3}>E_{5}$.

The simplest possible game is a one-move game, in which only $A$ gets a move. Suppose $A$ has probability $p$ of winning with this move; if he fails, with probability $1-p$, then his opponent $B$ wins. We ask, for what values of $p$ should a double be offered by $A$, and, if offered by $A$, for what values of $p$ should it be accepted by $B$ ? Clearly there is no value to exclusivity in $A$ 's right to double in this case.

Now if $A$ either cannot double or chooses not to double, he expects to win

$$
\begin{aligned}
E_{0}=E_{3}=E_{4}=E_{5} & =p .1+(1-p)(-1) \\
& =2 p-1 .
\end{aligned}
$$

If $A$ does double and $B$ accepts, then $A$ expects to win $2 E_{3}=4 p-2$. Thus $B$ should quit if $4 p-2>1$, that is, if $p>0.75$. If $p<0.75$, then $B$ will accept any double, and therefore $A$ should offer it whenever his expected profit is positive, that is, whenever $p>0.5$. 
The above one-move criteria ( 0.5 for doubling, 0.75 for quitting) are familiar to all backgammon players, but are often applied erroneously at earlier stages in the game. In summary, when both players are using the above optimal strategies, for a one-move game at probability $p$, the expected profit to player $A$ is

$$
E_{1}=E_{2}=\left\{\begin{aligned}
1, & p>0.75 \\
4 p-2, & 0.5<p<0.75, \\
2 p-1, & p<0.5
\end{aligned}\right.
$$

\section{Two-move games}

A general two-move game can be specified by two parameters $p$ and $q$, with $p$ being the probability that $A$ will win the game with his first move, and $q$ the probability that $B$ will win the game with his first move (assuming $B$ gets to make such a move). If both $A$ and $B$ fail with their first moves, the game is over, and $A$ has won.

First let us assume that there is no doubling at all in this game, either because it is prohibited, or because $A$ owns the exclusive right to the first double and chooses not to exercise it. Then, when $A$ moves, either (with probability $p$ ) $A$ wins $\$ 1$ immediately, or (with probability $1-p$ ) $B$ gets to move, in which case either (with probability $q$ ) $B$ wins $\$ 1$ or (with probability $1-q$ ) $A$ wins $\$ 1$. Thus the net expected profit to $A$ is

$$
\begin{aligned}
E_{0}=E_{5} & =p \cdot 1+(1-p)[q(-1)+(1-q)(1)] \\
& =1-2 q(1-p) .
\end{aligned}
$$

Next suppose that $A$ either cannot double, or chooses not to double, but $B$ may double if he wishes, should he get a move. Then, when $A$ moves, either (with probability $p$ ) $A$ wins $\$ 1$ immediately, or (with probability $1-p$ ), $B$ is presented with a one-move game at probability $q$. Thus, using the result $(2.4)$, we have

$$
\begin{aligned}
E_{3}= & E_{4}=p .1-(1-p) \cdot\left\{\begin{aligned}
1, & q>0.75, \\
4 q-2, & 0.5<q<0.75, \\
2 q-1, & q<0.5
\end{aligned}\right. \\
& =\left\{\begin{aligned}
2 p-1, & q>0.75, \\
p-2(1-p)(2 q-1), & 0.5<q<0.75, \\
p-(1-p)(2 q-1), & q<0.5 .
\end{aligned}\right.
\end{aligned}
$$

We now have all the information necessary to establish the optimal strategies. In the first place, suppose that a double has been legally offered by $A$. Then $B$ 
should accept if $2 E_{3}<1$, where $E_{3}$ is given by (3.2), that is, if $p<p_{Q}(q)$ where

$$
p_{Q}(q)=\left\{\begin{aligned}
0.75, & q>0.75 \\
\frac{8 q-3}{8 q-2}, & 0.5<q<0.75 \\
1-\frac{1}{4 q}, & 0.25<q<0.5 \\
0, & q<0.25
\end{aligned}\right.
$$

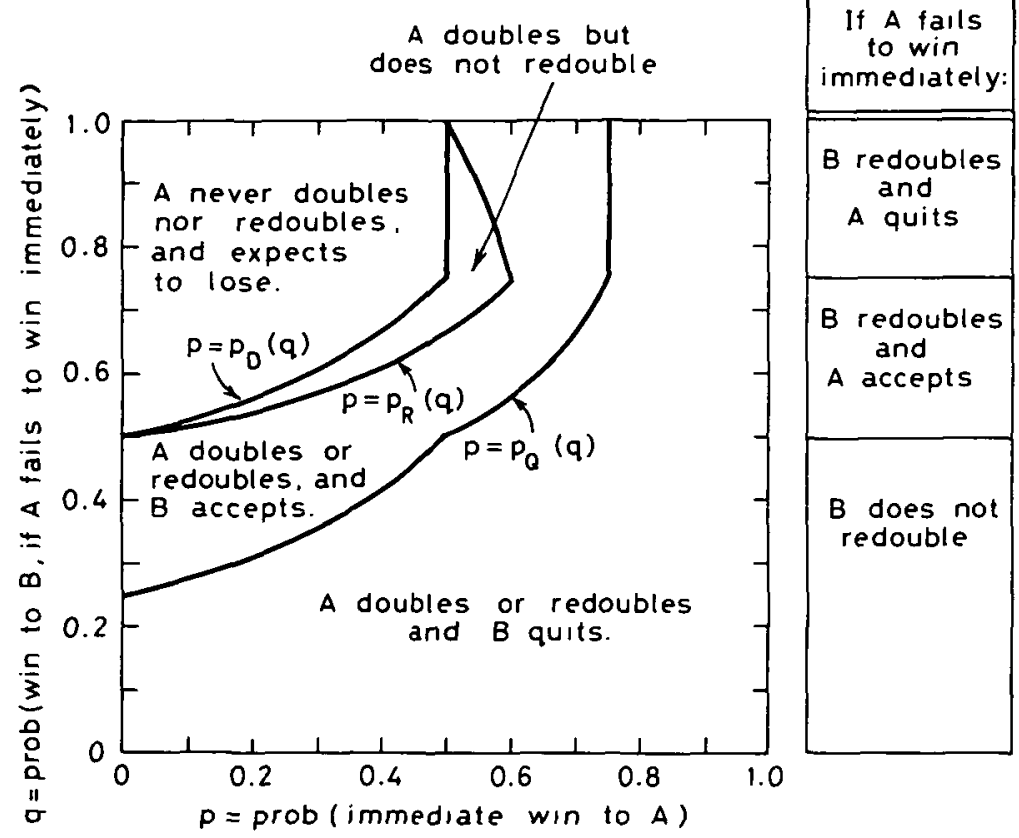

Fig. 1. Complete strategies for two-move games.

The curve $p=p_{Q}(q)$ is shown in Fig. 1 in the $(p, q)$ plane, $0 \leqslant p \leqslant 1,0 \leqslant q \leqslant 1$. Whenever the point $(p, q)$ lies to the right of this curve, $B$ should quit. When $q=1$ we have a one-move game, and $B$ quits if $p>0.75$; however, we now see that this one-move quitting strategy also applies unchanged to games with $0.75<q<1$ that $B$ is very likely but not certain to win, given a move. As soon as $B$ 's own move is less than $75 \%$ favourable, his quitting strategy becomes progressively more conservative as $q$ decreases until, when $q<0.25, B$ always quits, irrespective of $p$. This is to be expected, since his chances are necessarily worse than those in a onemove game at probability $1-q$, and we already know that he should quit that if $1-q>0.75$.

The point $p=q=0.75$ in Fig. 1 is interesting in that it represents a position (which can actually occur in backgammon) with a probability as high as 0.8125 
of an eventual win to $A$ in a game without doubling, and yet is borderline for acceptance of a double by $B$. In [2] it is shown that, in a "continuous" game, $B$ should quit whenever such a probability of an eventual win to $A$ exceeds 0.8 . However, it appears that, in discrete-move games, $B$ can accept in some such circumstances.

If $A$ owns the exclusive right to the next double, his optimal (redoubling) strategy is to redouble if $2 E_{3}>E_{5}$, that is, if $p>p_{R}(q)$ where

$$
p_{R}(q)=\left\{\begin{aligned}
\frac{3-2 q}{4-2 q}, & q>0.75 \\
\frac{6 q-3}{6 q-2}, & 0.5<q<0.75 \\
0, & q<0.5 .
\end{aligned}\right.
$$

The curve $p=p_{R}(q)$ is also shown on Fig. 1. The portion for $q>0.75$, with $0.5<p_{R}(q)<0.6$, is remarkable in that $p_{R}$ is a decreasing function of $q$. That is, in this range $A$ is the more likely to redouble, the less likely it is he will win! This is because in this range $A$ must quit if subsequently redoubled. If $q$ is not too large, $A$ 's chance of a final win, if the game is played out, is high enough to encourage him not to allow $B$ the option of a redouble. On the other hand, if $q$ is very high, $A$ will consider this chance too small to worry about, and will double, betting everything on his first throw.

Finally, $A$ 's non-exclusive doubling strategy is to double if $2 E_{3}>E_{4}$, that is (since $\left.E_{3}=E_{4}\right)$, if $E_{3}>0$, or $p>p_{D}(q)$ where

$$
p_{D}(q)=\left\{\begin{aligned}
0.5, & q<0.75, \\
\frac{4 q-2}{4 q-1}, & 0.5<q<0.75, \\
0, & q<0.5 .
\end{aligned}\right.
$$

The fact that $2 E_{3} \lessgtr 0$ for $p \lessgtr p_{D}(q)$ means that $A$ doubles whenever he expects to win the game. However, since $E_{0}\left(p_{D}(q), q\right)>0, A$ sometimes refrains from doubling in a game that he would expect to win if doubling were prohibited.

\section{Games of three or more moves}

As soon as the game may proceed to three or more moves, that is, if $A$ is not certain to win, given two moves, the analysis becomes very much more complicated than for the two-move case. No longer is it possible to classify exactly and uniquely all possible three-move games in terms of a finite number of input parameters such 
as $p$ and $q$ of Section 3. This is because, if $A$ 's first move does not win the game for him immediately, the probability of his winning with his second move (if he gets one) will change in accordance with the procedures for playing the particular game under consideration. The actual position arrived at by $A$ after his move, as distinct from the expected position estimated before that move, is what determines $B$ 's doubling decision when $B$ is about to move, and hence the expected result of the game.

In order to provide a rational framework to analyse a game with specified rules, we must define a set of positions which each player may hold in such a game. Then, if player $A$ has position $i$ and player $B$ has position $j$, the state of the game is completely characterized by $i$ and $j$, and the expected profits need to be determined as functions of $i$ and $j$, that is, $E_{n}=E_{n}(i, j), n=0$ to 5 .

We assume here that $i$ and $j$ take integer values, although there is no reason why a similar analysis cannot be carried out for a game with continuous evolvement of positions, as in [2]. It is convenient to take $i=0$ to mean that the game has already been won, and $i=1$ to mean that the game must be won at the next move. Generally, we expect that the positions improve as $i$ decreases, although this is not essential to the analysis.

Next, we must define a set of transition probabilities $\alpha_{i k}$ for these positions. That is, $\alpha_{i k}$ is the probability that a player having position $i$ before his move, will have position $k$ after that move. In fact the determination of what is the new state $k$ may be based on two factors, one of chance, and one of choice by the player of how to play the move. We assume that when such an element of choice exists, the optimal decision is always made, so that it is only the chance element that concerns us here.

We are also assuming that (i) the opposing player's position is unaffected by the current move, and (ii) both players operate under the same rules, that is, the same set of $\alpha_{i k}$. Both of these assumptions can be relaxed, but are valid for the non-contact backgammon end games of interest here. They are not valid for backgammon during the contact phase of the game. We assume also that $0 \leqslant k<i$, that is, that every move improves the position, although again this is not a necessary assumption. Whatever may be the range of $k$, we must of course have $\sum_{k} \alpha_{i k}=1$.

Note that the two-move games of Section 3 correspond to positions $i$ for player $A$ such that $\alpha_{i k}=0, k \geqslant 2$, with $p=\alpha_{i 0}=1-\alpha_{i 1}$. The opposing player $B$ can have any position $j$, but for him the only transition probability of significance can be $q=\alpha_{j 0}$, since $B$ can only ever make one move. A three-move game is one where player $B$ has $\alpha_{j k}=0, k \geqslant 2$, and player $A$ can have any position $i$. However, since in this case $A$ may make two moves, all transition probabilities $\alpha_{i k}$ for $A$ are significant.

Once the set of positions and transition probabilities are known, there is no 
difficulty in computing expected profits and optimal strategies, using the results

$$
\begin{aligned}
& E_{0}(i, j)=-\sum_{k=0}^{i-1} \alpha_{i k} E_{0}(j, k), \\
& E_{3}(i, j)=-\sum_{k=0}^{i-1} \alpha_{i k} E_{2}(j, k), \\
& E_{4}(i, j)=-\sum_{k=0}^{i-1} \alpha_{i k} E_{1}(j, k), \\
& E_{5}(i, j)=-\sum_{k=0}^{i-1} \alpha_{i k} E_{3}(j, k) .
\end{aligned}
$$

These equations simply express the fact that, after $A$ 's current move, $B$ will be presented with a game in one of the states $(j, k)$, the right to double being indicated by the subscript on each $E_{n}$, as in Section 2 .

Equations (4.2), (4.3) and (4.4) enable computation of the values of $E_{3}, E_{4}$ and $E_{5}$ when the game is in the state $(i, j)$, given values of $E_{1}, E_{2}$ and $E_{3}$ for all lower states. This then enables determination of the optimal strategies and, using (2.1), (2.2), of values for $E_{1}$ and $E_{2}$ at state $(i, j)$. Thus the complete game may be analysed recursively (that is, by "dynamic programming"), starting from known results for the lowest states.

In particular, for all $n$ and all $k>0$, we have $E_{n}(0, k)=E_{n}(1, k)=1$ and $E_{n}(k, 0)=-1$. The 1-move results $(2.3),(2.4)$ for the state $(i, 1)$ with $p=\alpha_{i 0}$, and the 2-move results for states $(i, j)$ such that $\alpha_{i k}=0, k \geqslant 2$, can now be re-derived by these methods, and we may then proceed to games with any number of moves.

\section{Application to backgammon}

Table 1 shows the 20 simplest backgammon positions and their transition probabilities $\alpha_{i k}$. The "description" of each position indicates the "point numbers" on which pieces lie. The positions are ranked here in order of decreasing $\alpha_{i 0}$, then $\alpha_{i 1}$, and so on, and every possible position with $\alpha_{i 0}>2 / 9$ is included here. In general, there is no guarantee that this particular numbering of positions correctly orders the strength of end positions in backgammon but, for the first 20 positions included here, it does so by any reasonable standard of measurement of "strength". As soon as we proceed further, there may be cause for argument regarding the right to the 21 st position, for example, between " $6-4$ " and " $1-1-1$ " (see [3]). For technical reasons, the positions "7", " 8 " and " 9 " are likely to occur only extremely rarely, but are nevertheless included here for completeness.

The question of optimal choice of move arises in only a few of the cases in 
TABLE 1

Transition probabilities for the 20 simplest backgammon positions

\begin{tabular}{rll}
\hline Position $i$ & Description & $\begin{array}{c}\text { Transition probabilities } \\
\left(36 \alpha_{i k}, k=0,1,2, \ldots\right)\end{array}$ \\
\hline 1 & "3" or better & 36 \\
2 & "4" or "3-1" & 34,2 \\
3 & "5" & 31,5 \\
4 & "4-1" & 29,7 \\
5 & "6" & 27,9 \\
6 & "2-2" & 26,10 \\
7 & "3-2" & 25,11 \\
8 & "5-1" or "4-2" & 23,13 \\
9 & "7" & $23,11,2$ \\
10 & "5-2" & $19,15,2$ \\
11 & "3-3" & 17,19 \\
12 & "4-3" & $17,17,2$ \\
13 & "8" & $17,14,3,2$ \\
14 & "6-1" & $15,19,2$ \\
15 & "5-3" & $14,17,3,2$ \\
16 & "6-2" & $13,18,3,2$ \\
17 & "9" & $12,15,4,3,0,2$ \\
18 & "4-4" & $11,20,3,0,2$ \\
19 & "6-3" & $10,17,4,3,0,2$ \\
20 & "5-4" & $10,17,4,3,0,0,0,0,2$ \\
& &
\end{tabular}

Table 1 , but will occur with increasing frequency for larger values of $i$. For example, holding position 10 ("5-2") and receiving the abominable dice throw of a "two-one", a foolish player might move to position 6 ("2-2") instead of position 2 ("4"). In constructing Table 1 we have always assumed that the best possible move is made. This of course requires us to have determined a correct ranking order of positions, for example, to be certain that position 2 is "stronger" than position 6. Fortunately, no such doubt exists for any of the positions listed, but as we go beyond positions listed, the decision on correct choice of move from some high-order positions may have to await determination of the optimal doubling strategies for all reachable lower-order positions, and therefore be built into the dynamic programming process.

Games $(i, j)$ in which $A$ holds one of the positions $i=1$ to 8 , or $i=11$, are two-move games, and the optimal strategies can be read off immediately from Fig. 1, using $p=\alpha_{i 0}, q=\alpha_{j 0}$. The only other positions for $A$ that result in twomove games are "1-1-1", "1-1-2" and "1-1-1-1", which have $p=\frac{1}{6}$ (any doublet wins immediately), and seven other 3- and 4-piece positions, which have $p=\frac{5}{36}$ (needing doublet-2 or better for an immediate win).

The next category includes positions 9, 10,12 and 14 (and some 3- and 4-piece positions not in Table 1) that suffer a 1 in 324 chance of leading to 3 moves, that 
is, by throwing "two-one" twice in a row. This is such an unlikely event that Fig. 1 still yields the correct strategy in almost all such cases. An exception is when $A$ holds position 12 ("4-3") and $B$ holds either position 11 ("3-3") or 12 . If we apply the two-move analysis (that is, in effect, assume that position 12 is identical to position 11) we find that $2 E_{3}=\frac{325}{324}=1.0031$, that is, $B$ should decline $A$ 's double. However, if we take correct account of the non-zero transition probability $\alpha_{12,2}$, we find from equation (4.2) that $2 E_{3}=\frac{1453}{1458}=0.9966$, and the double should be accepted. It is, however, obvious that these particular decisions are by extremely narrow margins, and a backgammon player who makes the "wrong" decision will suffer a negligible penalty.

Position 10 ("5-2") is perhaps the most interesting of all end positions in backgammon. It is in the above category of almost-certain two-move positions, for which Fig. 1 always indicates the correct strategy, and is the only position in backgammon with $0.5<\alpha_{i 0}<0.6$. That is, position 10 is the only position for $A$ allowing a redouble to be offered against some of $B$ 's positions, that would not be offered if $B$ had a worse position. In fact the only such positions for $B$ are positions 1 and 2 ; the doubling cube is retained against positions 3 to 7 , and offered again against all positions inferior to 7 . Position 10 is also interesting when held by $B$, as it forms the borderline for offering a double for many positions for $A$.

No position in Table 1 fails to produce a win to the player holding it if he is given three moves, and the full expectations and strategies can be evaluated by hand for all games involving them. However, the equations can easily be programmed for computer use, and results have been obtained on a TRS-80 minicomputer for all games involving these 20 positions, and for about 30 more.

Table 2 summarizes the optimal strategies for the first 20 positions. A " $d$ " indicates a double but not a redouble, and an " $A$ " a double or a redouble, which should be accepted in both cases. A blank in the upper right corner indicates that $A$ doubles and $B$ quits, whereas a blank in the lower left corner indicates that $A$ should not double. Positions 1 to 5 for $A$ are not included since, if $A$ holds these positions, he should double or redouble, and $B$ should quit with any position, although the quitting decision is exactly borderline when $B$ holds positions 1 to 5 against position 5 . Thus the game is over as soon as $A$ reaches one of these positions.

A somewhat larger table of strategies is given in [3], based on an approximate theory. In fact this approximate theory yields identical strategies for these first 20 positions, and only slight and minor changes in strategies for some higher-order positions.

An example of a much higher-order state analysed according to the present (exact) theory is the position "6-6" versus the position " $5-3-3$ ". The computed value of $2 \mathrm{E}_{3}$ is 0.999968 , and therefore a double should be accepted, so losing $B$ three cents less than he would lose by quitting, in a game with a stake of a thousand dollars! The results confirm the principle ([1], page 70$)$ that a double should always 


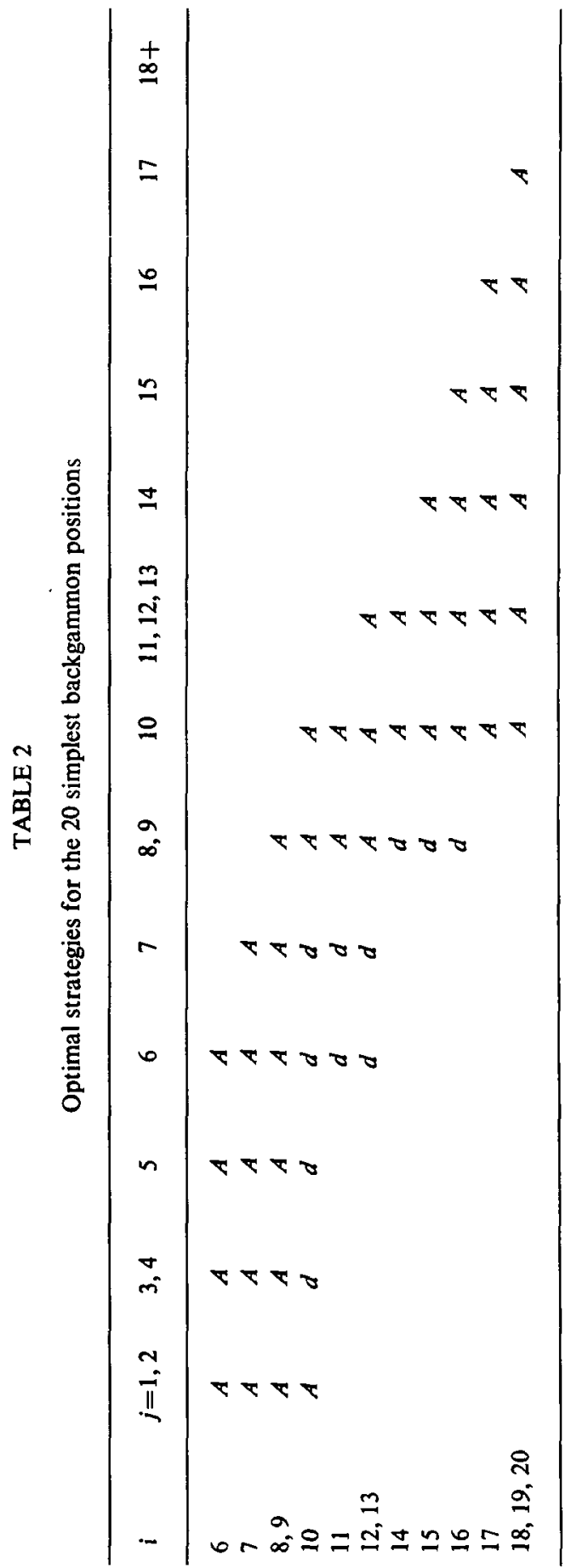


be offered by a player holding just two pieces in his home board against three or more by his opponent, and that such a double should always be declined unless the doubler holds " $6-6$ ". If $A$ holds " $6-6$ ", acceptance of his double is warranted with many three-piece positions for $B$, the position " $5-3-3$ " above providing the nearest to a borderline case, and the position " $6-3-3$ " being just on the quitting side.

\section{Acknowledgements}

Valuable critical comments by Bill Henderson, Alf van der Poorten and the referees are gratefully acknowledged.

\section{References}

[1] O. Jacoby and J. R. Crawford, The backgammon book (Pan, London, 1975).

[2] E. B. Keeler and J. Spencer, "Optimal doubling in backgammon", Operations Research 23 (1975), 1063-1071.

[3] E. O. Tuck, "Backgammon end-game doubling strategies", University of Adelaide, Applied Mathematics Report T7904 (1979).

Department of Applied Mathematics

University of Adelaide

Adelaide

South Australia 5001 